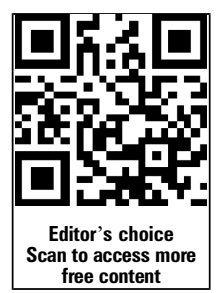

can to access mo free content

\title{
Nerve ultrasound depicts peripheral nerve enlargement in patients with genetically distinct Charcot-Marie-Tooth disease
}

${ }^{1}$ Department of Neurology, Graduate School of Medical Science, Kyoto Prefectural University of Medicine, Kyoto, Japan

${ }^{2}$ Department of Medical Education and Primary Care, Graduate School of Medicine, Kyoto Prefectural University of Medicine, Kyoto, Japan ${ }^{3}$ Department of Neurology and Geriatrics, Kagoshima University Graduate School of Medical and Dental Sciences, Kagoshima, Japan

${ }^{4}$ North Medical Center, Kyoto Prefectural University of Medicine, Kyoto, Japan

Correspondence to Dr Yu-ichi Noto, Department of Neurology, Kyoto Prefectural University of Medicine Graduate School of Medical Science, 465 Kajii-cho, Kamigyo-ku, Kyoto 602-0841, Japan; y-noto@koto.kpu-m.ac.jp

Received 31 March 2014 Revised 6 July 2014 Accepted 13 July 2014 Published Online First 4 August 2014

\section{SLinked}

- http://dx.doi.org/10.1136/ jnnp-2014-308855

\section{CrossMark}

To cite: Noto $Y$, Shiga $K$, Tsuji Y, et al. J Neurol Neurosurg Psychiatry 2015:86:378-384.

\author{
Yu-ichi Noto, ${ }^{1}$ Kensuke Shiga, ${ }_{1}^{2}$ Yukiko Tsuji, ${ }_{1}^{1}$ Ikuko Mizuta, ${ }^{1}$ Yujiro Higuchi, ${ }^{3}$ \\ Akihiro Hashiguchi, ${ }^{3}$ Hiroshi Takashima, ${ }^{3}$ Masanori Nakagawa, ${ }^{4}$ Toshiki Mizuno $^{1}$
}

\begin{abstract}
Objective To elucidate the ultrasound (US) features of peripheral nerves including nerve roots in patients with different types of Charcot-Marie-Tooth disease (CMT), and the association between US findings, clinical features and parameters of nerve conduction studies (NCS) in CMT1A.
\end{abstract}

Methods US of median, sural and great auricular nerves and the $\mathrm{C} 6$ nerve root was performed in patients with CMT1A ( $n=20), M P Z$-associated CMT $(n=3)$, NEFLassociated CMT $(\mathrm{n}=4)$, EGR2-associated CMT $(\mathrm{n}=1)$, ARHGEF10-associated CMT $(\mathrm{n}=1)$ and in controls $(n=30)$. In patients with CMT1A, we analysed the correlations between US findings and the following parameters: age, CMT Neuropathy Score (CMTNS) and NCS indices of the median nerve.

Results Cross-sectional areas (CSAs) of all the nerves were significantly increased in patients with CMT1A compared with that in controls. In MPZ-associated CMT, increased CSAs were found in the median nerve at wrist and in the great auricular nerve, whereas it was not increased in patients with NEFL-associated CMT. In patients with CMT1A, there was a positive correlation between CMTNS and the CSAs in the median nerves or great auricular nerves. In median nerves in patients with CMT1A, we found a negative correlation between the nerve conduction velocity and the CSA.

Conclusions Nerve US may aid in differentiating among the subtypes of CMT in combination with NCS. In CMT1A, the median nerve CSA correlates with the disease severity and peripheral nerve function.

\section{INTRODUCTION}

Charcot-Marie-Tooth disease (CMT) is a clinically and genetically heterogeneous inherited neuropathy, characterised by distal muscle atrophy, weakness and sensory loss with reduced tendon reflexes. Nerve conduction studies (NCS) differentiate CMT into the demyelinating type (median nerve motor conduction velocity $(\mathrm{MCV})<38 \mathrm{~m} / \mathrm{s}$ ) and axonal type (median nerve $\mathrm{MCV}>38 \mathrm{~m} / \mathrm{s}$ ). ${ }^{1}$ Autosomal dominant (AD) demyelinating (CMT1), AD axonal (CMT2), autosomal recessive (AR; CMT4) and X linked (CMTX) forms of CMT exist. The main pathology of CMT4 is demyelinating. CMTX type 1 (CMT1X) is the second most common form of CMT. Most males with CMT1X have intermediately slow MCV between 30 and $45 \mathrm{~m} / \mathrm{s}$, and the pathology of CMT1X is axonal loss and some segmental demyelination. ${ }^{2}$ Over the last decade, there have been rapid advances in identifying genetic abnormalities in patients with CMT. More than 45 different CMT-causing genes have been described. ${ }^{3}$ Furthermore, Hattori et $a l^{4}$ reported that patients with MPZ, PMP2 and Cx32 mutations present both demyelinating and axonal types.

High-resolution ultrasound (US) has been increasingly used for the non-invasive assessment of peripheral nerve diseases. ${ }^{5}{ }^{6}$ US features of some CMT subtypes have been reported. ${ }^{7}{ }^{8}$ Schreiber et $a l^{9}$ reported direct comparisons of nerve US findings between CMT subtypes and the correlation between nerve US indices and NCS parameters. However, detailed studies, including those on US assessment of nerve roots and clarification of the correlation between US findings and the disease severity, remain limited.

The purpose of this study was to describe US features of peripheral nerves including C6 nerve root in different types of CMT, and analyse the correlation between US findings and clinical/neurophysiological parameters.

\section{METHODS}

The study was conducted at Kyoto Prefectural University of Medicine Hospital between January and November 2012. Informed consent was provided by each participant, and the study protocol was conducted in accordance with the Declaration of Helsinki.

\section{Subjects}

We examined 35 consecutive patients (21 males and 14 females; age range $10-80$ years; mean \pm SD $46.7 \pm 19$ years) with hereditary motor and sensory neuropathy, 4 of whom were blood relatives to at least one other patient of the study group. In all patients, the diagnosis was based on the results of NCS and a family history of the disease.

Thirty sex-matched and age-matched controls (19 males and 11 females; age range $24-84$ years; mean \pm SD $42.7 \pm 16$ years) were recruited from the staff of Kyoto Prefectural University of Medicine and their families, who were free of any neuromuscular symptoms (eg, numbness and tingling or weakness of limbs), diabetes mellitus and alcoholism.

\section{Genetic testing}

First, we investigated whether patients with the demyelinating type of CMT have PMP22 duplication or deletion by fluorescence in situ hybridisation. For patients with the demyelinating type of CMT who 
had no PMP22 rearrangement or patients with the axonal type of CMT, genomic DNA was extracted from their peripheral blood leucocytes, and then 30 disease-causing genes related to CMT were screened for using the custom MyGeneChip CustomSeq Resequencing Array (Affymetrix, Inc, Santa Clara, California, USA), which was designed to screen for CMT and related diseases, such as ataxia with oculomotor apraxia types 1 and 2, spinocerebellar ataxia with axonal neuropathy and distal hereditary motor neuropathy. ${ }^{10}$ We designed 363 primer sets to include the entire coding regions and flanking sequences of the 30 genes (box 1). When a novel mutation was detected, we performed familial segregation analysis to elucidate the pathogenicity of the mutation if possible.

\section{Ultrasound}

All US examinations were performed by the same examiner (Y-iN) trained in neuromuscular US, using a GE Logic P5 System (GE Healthcare Japan, Tokyo, Japan) with a $12 \mathrm{MHz}$ linear-array probe. The cross-sectional areas (CSAs) of the following nerves and nerve roots were measured: median nerve, sural nerve, great auricular nerves and C6 nerve root. Additionally, the diameter of the C6 root was measured. The median and the sural nerves were selected for evaluation because those nerves have been frequently evaluated in preceding studies in patients with CMT. The greater auricular nerve was examined because of the unique travelling course in the neck surface and its easy accessibility. The examiner (Y-iN) was not blinded to the diagnosis or clinical or electrophysiological findings. All participants were placed in a supine position when

\section{Box 1 Genes analysed in the screening}

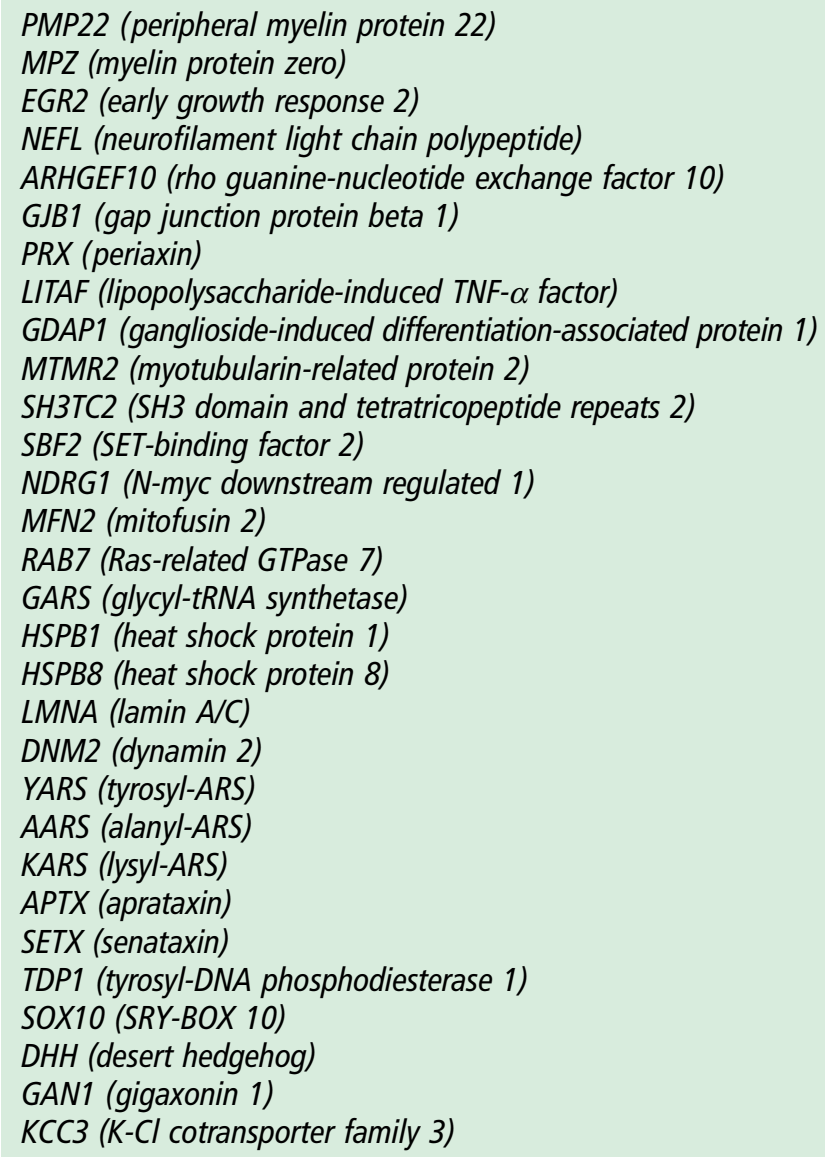

their median nerves and cervical nerve roots were examined, and in a prone position when their sural nerves were examined. The median nerve was imaged at the wrist crease, in the middle of the forearm, and in the middle of the upper arm. The sural nerve was imaged at $10 \mathrm{~cm}$ proximal to the lateral malleolus. We used the saphenous vein as a landmark when we identified the sural nerve beside the vein. The great auricular nerve was imaged at the midpoint between the top of the sternum and mandibular angle. We could identify the nerve in front of the sternocleidomastoid muscle (figure 1A). The CSAs were calculated by manual tracking of the nerve circumference including the hyperechoic rim. The diameter of the root was measured between the outer surfaces of the hyperechoic rims. The measured site of the $\mathrm{C} 6$ nerve root was about $1 \mathrm{~cm}$ distal to the transverse process after identifying the $\mathrm{C} 6$ vertebra using a previously reported procedure (figure $1 \mathrm{~B}) .{ }^{11}$

\section{Nerve conduction studies}

Using standard techniques (Neuropack EMG system (Nihon Kohden, Tokyo, Japan)), conventional NCS was performed. The skin temperature was maintained above $32^{\circ} \mathrm{C}$. The distal motor latency (DML), compound muscle action potential (CMAP) amplitude and MCV were recorded from the median nerve. The MCV was assessed in the wrist to elbow. The sensory nerve action potential (SNAP) amplitude, SNAP duration and sensory conduction velocity (SCV) were recorded from median and sural nerves. Antidromic median and sural nerve SNAPs were recorded from digit II and behind the lateral malleolus, respectively. We analysed the corresponding nerves in unilateral side using US and NCS.

\section{Clinical assessment}

Patients with CMT underwent clinical and neurophysiological assessment based on the CMT Neuropathy Score (CMTNS). ${ }^{12}$ The CMTNS is composed of nine items: sensory symptoms, motor symptoms of legs and arms, pin sensibility, vibration, strength of legs and arms, ulnar CMAP amplitude and ulnar SNAP amplitude. The CMTNS ranges from 0 (no deficit) to 36 (maximal deficit).

\section{Statistics}

In the analysis of NCS parameters, if no CMAP and SNAP responses were elicited, they were excluded from analysis except for the amplitude data (CMAP and SNAP amplitudes in no response were regarded as $0.001 \mathrm{mV}$ and $0.001 \mu \mathrm{V}$, respectively). Fisher's exact test was used to analyse the gender ratio between patients with CMT and controls. To compare CMTNS between the different CMT subgroups, and the US parameters (CSA and diameter) among the different CMT subgroups and controls, a Bonferroni-corrected Mann-Whitney U test was applied. The correlation between the US findings (CSA and diameter) and clinical parameters (age, height, weight, body mass index and CMTNS) or the electrophysiological parameters (DML, MCV, SCV, CMAP amplitude and SNAP amplitude) in controls and patients with CMT1A was tested with Pearson correlation coefficients. In all comparisons, a $\mathrm{p}$ value of less than 0.05 was considered significant. All statistical analyses were performed using STATA software (Stata Corp, Texas, USA).

\section{RESULTS}

\section{Clinical data and CMTNS}

On the basis of the genetic testing results, 20 patients were classified with PMP 22 duplication-associated CMT (CMT1A), 3 with MPZ-associated CMT (2 CMT1B and 1 CMT2J), 4 with 


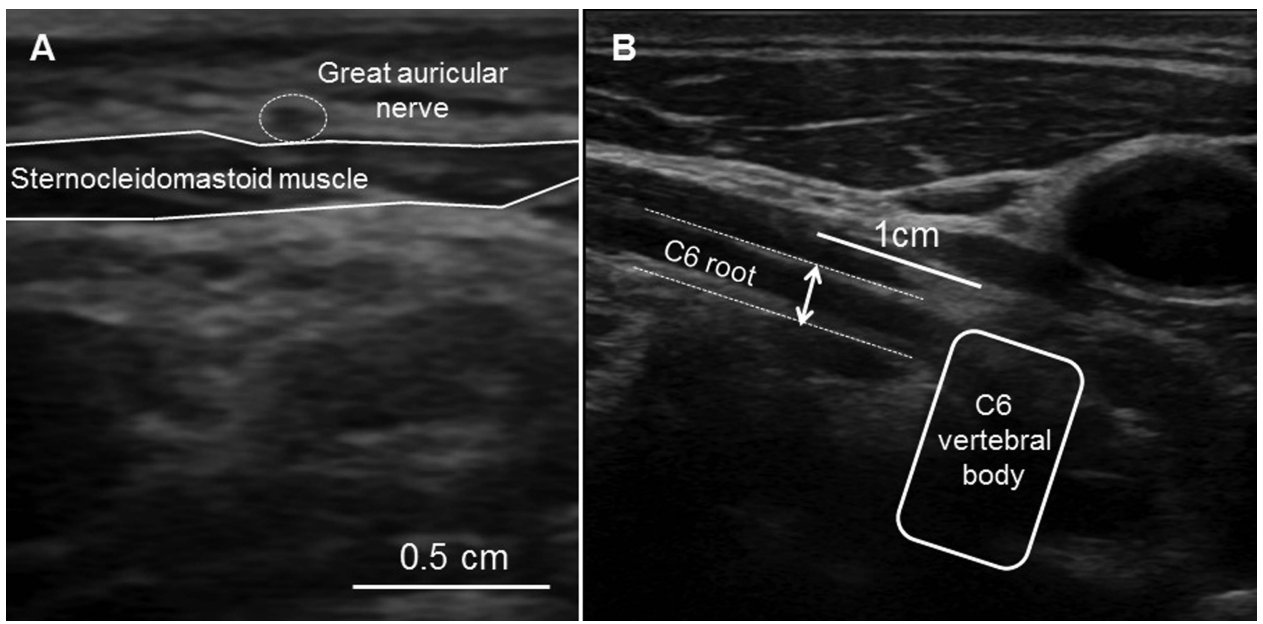

Figure 1 The ultrasound images of the great auricular nerve $(A)$ and the measured site in the $C 6$ nerve root (B). The dotted circle indicates the cross-sectional image of the great auricular nerve (A). The distances between the outer surfaces of the hyperechoic rims (between arrowheads) were measured as the nerve root diameter (B).

NEFL-associated CMT (2 CMT1F and 2 CMT2E), 1 with EGR2-associated CMT and 1 with ARHGEF10-associated CMT. The patient with CMT2J presented hearing loss and autonomic dysfunction such as Adie's pupils and dysuria, in addition to the distal dominant muscle weakness. All of the patients with NEFL-associated CMT showed distal dominant muscle weakness. The patient with EGR2-associated CMT was reported by us recently. ${ }^{13}$ The patient with ARHGEF10-associated CMT presented muscle weakness in lower extremities with a slight decrease in vibratory sensation. Direct sequencing of the ARHGEF10 gene in the patient with ARHGEF10-associated CMT revealed a heterozygous single nucleotide substitution, c.2435T $>$ C, which might be a novel mutation. We could confirm the same mutation in the proband's brother with similar symptoms and electrophysiological findings, although gene analysis of other asymptomatic family members was not possible. No pathogenic mutation was identified in three patients with demyelinating type CMT and three with axonal type CMT.

Demographic data, the electrophysiological neuropathy type, CMTNS and gene mutation of each CMT subtype are shown in table 1. No significant difference in CMTNS was demonstrated among the CMT subtypes. In groups with MPZ or NEFL mutation, demyelinating and axonal types were mixed.

\section{US findings}

US findings in each CMT group and the control group are presented in table 2 and figure 2. The CSAs in patients with CMT1A were larger than those in controls irrespective of examination sites (figure 2). Although all mean CSAs and the C6 root diameter in patients with MPZ mutation tended to be larger than in controls, significant differences existed in the median nerve CSA at wrist and in the great auricular nerve CSA. There were no significant differences between all CSAs in patients with NEFL mutation and controls, whereas median nerve CSAs at three sites in patients with CMT1A were larger than in patients with NEFL mutation including the demyelinating type. In a patient with EGR2 mutation, CSAs of proximal sites tended to be large, and the C6 root CSA in a patient with EGR2 mutation was larger than the mean CSA value +2 SDs in controls. Although we could not identify the $\mathrm{C} 6$ root and great auricular nerve in a patient with ARHGEF10 mutation, CSAs of the median nerve and sural nerve in the patient were slightly larger than the mean CSAs of controls.

\section{Nerve conduction studies}

Results of parameters of the NCS on the median and the sural nerves are listed in table 3. In patients with CMT1A, motor and

Table 1 Biometric data, electrophysiological neuropathy types, CMT Neuropathy Score and gene mutations

\begin{tabular}{|c|c|c|c|c|c|c|}
\hline & $\begin{array}{l}\text { PMP22 duplication } \\
(\mathrm{n}=20)\end{array}$ & $\begin{array}{l}\text { MPZ mutation } \\
(\mathrm{n}=3)\end{array}$ & $\begin{array}{l}\text { EFL mutation } \\
(n=4)\end{array}$ & $\begin{array}{l}\text { EGR2 mutation } \\
(\mathrm{n}=1)\end{array}$ & $\begin{array}{l}\text { ARHGEF10 mutation } \\
(\mathrm{n}=1)\end{array}$ & $\begin{array}{l}\text { Controls } \\
(n=30)\end{array}$ \\
\hline Age, mean (range) & $47.6(21-78)$ & $39.7(10-69)$ & $47.3(27-68)$ & 49 & 67 & $42.7(24-84)$ \\
\hline Gender (M/F) & $10 / 10$ & $2 / 1$ & $2 / 2$ & $0 / 1$ & $1 / 0$ & $19 / 11$ \\
\hline Height $(\mathrm{cm})$, mean $(\mathrm{SD})$ & $161.9(10.0)$ & $152.7(16.1)$ & $168.4(5.1)$ & 160.0 (NA) & 173.5 (NA) & $162.8(11.5)$ \\
\hline Weight $(\mathrm{kg})$, mean (SD) & $58.2(11.1)$ & $55.6(17.5)$ & $63.5(22.2)$ & $56.0(\mathrm{NA})$ & 76.0 (NA) & $58.4(10.8)$ \\
\hline Body mass index, mean(SD) & $22.1(3.3)$ & $23.4(2.5)$ & $22.3(7.6)$ & 23.8 (NA) & 25.2 (NA) & $21.9(2.2)$ \\
\hline Demyelinating type/axonal type & $20 / 0$ & $2 / 1$ & $2 / 2$ & $1 / 0$ & $0 / 1$ & NA \\
\hline CMT Neuropathy Score, mean (range) & $14.0(7-28)$ & $12.0(10-14)$ & $15.5(9-25)$ & 7.0 & 7.0 & NA \\
\hline Gene mutations & PMP22 duplication & $\begin{array}{l}\text { CMT1B: } \\
\text { Tyr68Cys } \\
(\mathrm{n}=2) ; \\
\text { CMT2J: } \\
\text { Thr124Met }\end{array}$ & $\begin{array}{l}\text { CMT1F: } \\
\text { Pro8Leu } \\
\text { (n=2) } \\
\text { CMT2E: } \\
\text { Glu396Lys; } \\
\text { Tyr389Cys }\end{array}$ & Thr387Asn & Thr109lle & NA \\
\hline
\end{tabular}

CMT, Charcot-Marie-Tooth disease; F, female; M, male; NA, not applicable. 
Table 2 Ultrasound findings in patients with Charcot-Marie-Tooth disease and controls

\begin{tabular}{|c|c|c|c|c|c|c|c|c|c|c|c|c|}
\hline & \multicolumn{2}{|c|}{$\begin{array}{l}\text { PMP22 } \\
\text { duplication }(n=20)\end{array}$} & \multicolumn{2}{|c|}{$\begin{array}{l}\text { MPZ mutation } \\
(\mathrm{n}=3)\end{array}$} & \multicolumn{2}{|c|}{$\begin{array}{l}\text { NEFL mutation } \\
(\mathrm{n}=4)\end{array}$} & \multicolumn{2}{|c|}{$\begin{array}{l}\text { EGR2 mutation } \\
(n=1)\end{array}$} & \multicolumn{2}{|c|}{$\begin{array}{l}\text { ARHGEF10 } \\
\text { mutation }(n=1)\end{array}$} & \multicolumn{2}{|c|}{ Controls $(n=30)$} \\
\hline & Mean (SD) & (n) & Mean (SD) & (n) & Mean (SD) & (n) & Mean (SD) & (n) & Mean (SD) & (n) & Mean (SD) & (n) \\
\hline \multicolumn{13}{|l|}{ Cross sectional area $\left(\mathrm{mm}^{2}\right)$} \\
\hline Median nerve (wrist) & $23.5(4.0)$ & (20) & $21.0(4.5)$ & (3) & $12.0(3.0)$ & (4) & 20.5 & (1) & 22.8 & (1) & $14.1(2.6)$ & (30) \\
\hline Median nerve (forearm) & $22.1(9.2)$ & (20) & $17.5(8.9)$ & (3) & $8.5(3.1)$ & (4) & 12.2 & (1) & 18.3 & (1) & $8.7(1.3)$ & (30) \\
\hline Median nerve (upper arm) & $42.4(11.8)$ & (20) & $28.9(12.3)$ & (3) & $18.2(4.4)$ & (4) & 34.5 & (1) & 36.6 & (1) & $16.5(2.7)$ & (30) \\
\hline C6 root & $29.8(10.7)$ & (12) & 17.0 & (1) & 17.1 & (1) & 42.2 & (1) & NA & (0) & $13.0(3.1)$ & (22) \\
\hline Great auricular nerve & $3.9(1.6)$ & (19) & $5.2(3.8)$ & (2) & 2.0 & (1) & 3.5 & (1) & NA & (0) & $1.7(0.6)$ & (25) \\
\hline Sural nerve & $11.0(4.8)$ & (20) & $7.5(2.5)$ & (3) & $6.0(2.5)$ & (4) & 6.7 & (1) & 14.3 & (1) & $5.8(1.5)$ & (29) \\
\hline \multicolumn{13}{|l|}{ Diameter (mm) } \\
\hline C6 root & $5.3(1.1)$ & (13) & 4.4 & (1) & 4.1 & (1) & 5.5 & (1) & NA & (0) & $3.6(0.5)$ & (25) \\
\hline
\end{tabular}

sensory conduction velocities reduced with decreased CMAP and SNAP amplitude. In patients with MPZ mutation, the patients with CMT1B had a very slow MCV, whereas the patient with CMT2J showed a nearly normal MCV. In patients with NEFL-mutations, the difference between CMT1F and 2E was similar to that between CMT1B and 2J. The patient with EGR2-associated CMT (CMT1D) showed a demyelinating pattern. The MCV and SCV were moderately slowed in the patient with ARHGEF10 mutation.
Correlation between US findings and clinical/ electrophysiological parameters in patients with CMT1A We analysed the correlation between US findings (nerve CSAs and C6 diameter) and clinical data (CMTNS, age, height, weight and body mass index)/electrophysiological parameters in patients with CMT1A. The CMTNS in patients with CMT1A was positively correlated with the CSA of the great auricular nerve and that of the median nerve at the upper arm (figure 3A, B). Moreover, an inverse association was noted between the C6 root CSAs and age
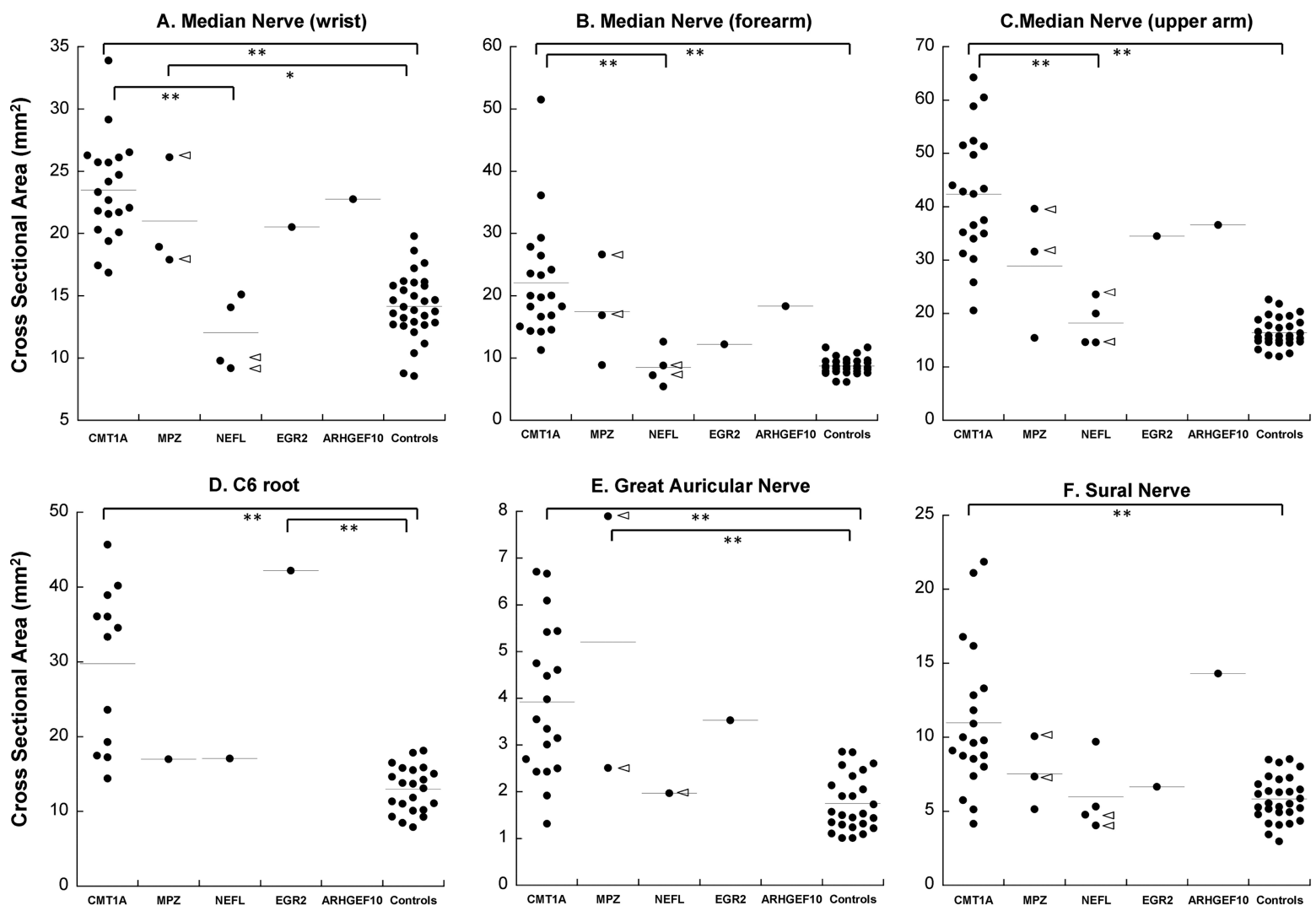

Figure 2 Ultrasound data on the median nerve, C6 root, great auricular nerve and sural nerve in patients with Charcot-Marie-Tooth disease (CMT) and controls. Horizontal bars indicate means. ${ }^{*} \mathrm{p}<0.05 ;{ }^{* *} \mathrm{p}<0.01$. Arrowheads indicate demyelinating type in patients with $M P Z$-associated and NEFL-associated CMT. Cross-sectional areas of the C6 root and great auricular nerve in patients with ARHGEF10-associated CMT were not recorded because of technical difficulty in ultrasound examination. 
Table 3 Nerve conduction study results

\begin{tabular}{|c|c|c|c|c|c|c|c|}
\hline & \multicolumn{3}{|c|}{ Median nerve (motor) } & \multicolumn{2}{|c|}{ Median nerve (sensory) } & \multicolumn{2}{|l|}{ Sural nerve } \\
\hline & DML (ms) & Amplitude (mV) & $\operatorname{MCV}(\mathrm{m} / \mathrm{s})$ & Amplitude $(\mu \mathrm{V})$ & $\mathrm{SCV}(\mathrm{m} / \mathrm{s})$ & Amplitude $(\mu \mathrm{V})$ & $\mathrm{SCV}(\mathrm{m} / \mathrm{s})$ \\
\hline PMP22 duplication $(\mathrm{n}=20)$ & 10.2 & 3.8 & 21.8 & 1.3 & 20.9 & 0.6 & 22.9 \\
\hline \multicolumn{8}{|l|}{$M P Z$ mutation $(n=3)$} \\
\hline CMT1B $(n=2)$ & 6.9 & 4 & 14.6 & 0.0 & NA & 0.0 & NA \\
\hline CMT2J $(n=1)$ & 3.7 & 11.3 & 46.4 & 9.7 & 49.4 & 6 & 51.9 \\
\hline \multicolumn{8}{|l|}{ NEFL mutation $(n=4)$} \\
\hline CMT1F $(n=2)$ & 8.0 & 2.4 & 24.2 & 0.0 & NA & 0.0 & NA \\
\hline CMT2E $(n=2)$ & 5.7 & 9.8 & 50.4 & 6.8 & 43.5 & 1.3 & 54.9 \\
\hline EGR2 mutation $(\mathrm{n}=1)$ & 6 & 4.7 & 23.2 & 2.1 & 26.8 & NE & NA \\
\hline ARHGEF10 mutation $(\mathrm{n}=1)$ & 4.7 & 5.6 & 41.7 & 14.7 & 40.2 & 6.5 & 48.8 \\
\hline
\end{tabular}

(figure 3C). Although statistically significant differences were not demonstrated, age tends to correlate inversely with CSAs of the median nerve at the fore arm, that at upper arm, the sural nerve and the great auricular nerve, whereas a positive relationship between age and CSAs of median nerve at the wrist was observed $(p=0.23)$. All clinical data except for the CMTNS and age showed no correlation with CSAs and the C6 diameter.

In the analysis between US findings of the median nerve and electrophysiological parameters, there was a significant negative correlation between the CSA at the forearm and MCV of the median nerve (between the wrist and elbow; $\mathrm{p}<0.05$; figure 4A). Likewise the CSA at the upper arm of the median nerve was negatively correlated with the MCV of the median nerve (between the wrist and elbow; $p<0.01$; figure $4 \mathrm{~B}$ ). No correlation was observed between the CSA and CMAP amplitude/ SNAP amplitude of the median nerve. Analysis of the correlation between US and electrophysiological findings of the sural nerve was not performed because SNAPs were not evoked in 18 of the 20 patients with CMT1A.

\section{DISCUSSION}

In this study, we confirmed that patients with CMT1A showed a uniform enlargement of nerves, including the nerve root, based on US imaging. Although small in number, we showed increased CSA in median nerves in individuals with MPZ mutations (CMT1B and 2J), EGR2 mutations (CMT1D) and ARHGEF10 mutations. In patients with NEFL mutations (CMT1F and 2E), however, the CSAs in the examined nerves are comparable to those in normal controls. This is the first report regarding the US findings in patients with CMT2J, 1F, 2E, 1D and ARHGEF10-associated CMT. The limitation of this study included a small number of patients with rare mutations. Therefore, these findings should be confirmed in a larger cohort in the future. Furthermore, we revealed not only the presence of a correlation between the CSAs and electrophysiological parameters, but also a correlation between the CSAs and clinical parameters (CMTNS) in patients with CMT1A.

In agreement with previous reports, we found markedly increased CSAs in all nerves and nerve roots in patients with CMT1A. ${ }^{6-9} 14$ The ranges of CSAs in great auricular and sural nerves of patients with CMT1A and controls overlapped to some extent (figure 2). Measuring CSAs in the median nerve and nerve root may facilitate a clear distinction among CMT1A, NEFL-associated CMT and a healthy state. Pazzaglia et $a l^{15}$ reported that the sural nerve CSA was not increased in the majority of patients with CMT1A (70\%). In our study, however, the sural nerve CSA in patients with CMT1A was significantly larger than that in controls. One of the factors influencing the difference between our results and the aforementioned study might be that CSAs were calculated by tracking the nerve circumference including the hyperechoic rim in that study. We measured CSAs by tracking the outline of the hyperechoic rim in consideration of the possibility that the nerve stroma including the epineurium proliferates in some subtypes of CMT. Robaglia-Schlupp et al ${ }^{16}$ reported that PMP22 overexpression enhanced collagen synthesis by fibroblasts, and noted the possibility that structures other than Schwann cells were affected in CMT1A.

In this study, three patients with MPZ mutations were included. Two of them were diagnosed with CMT1B. The remaining patient was diagnosed with CMT2J. There have been no reports including US findings in patients with CMT2J. CSAs in all nerves of the patient with CMT2J were the smallest in the three patients with MPZ-associated CMT, and these, excluding the median nerve (wrist) of the patient with CMT2J, were nearly the same as the mean values of the control group. Median nerve CSAs of the other two patients with demyelinating-type MPZ-associated CMT (CMT1B) tended to be larger than in controls (figure 2). These findings are consistent with a previous study on CMT1B. ${ }^{8}$

This is the first report on nerve US findings including patients with NEFL-associated CMT. Four patients with NEFLassociated CMT were examined in this study, comprising two with CMT1F and two with CMT2E. Although two of the four patients had demyelinating-type CMT, they did not show the enlargement of peripheral nerves. The NEFL gene encodes the neurofilament light chain polypeptide (NEFL), which is one of the most abundant cytoskeletal components of neurons, and plays a pivotal role in the assembly and maintenance of the axonal cytoskeleton. Fabrizi et $a l^{17}$ noted that the main pathological finding in patients with NEFL-associated CMT was axonopathy with marked structural alterations in the cytoskeleton and significant secondary demyelination. It appears that nerve conduction velocity slowing in NEFL-associated CMT is associated with mutations affecting the NEFL protein head domain. ${ }^{18}$ From these findings, patients with demyelinating type CMT may not always present increased CSAs of nerves, although previous studies have reported that patients with other demyelinating type CMT generally showed increased CSAs of nerves. ${ }^{9} 19$

The patient with EGR2-associated CMT in this study presented with a mild, demyelinating, adult-onset form. ${ }^{13}$ The EGR2 gene encodes early growth response-2 protein (EGR2), which plays a role in peripheral nerve myelin development and maintenance, and 

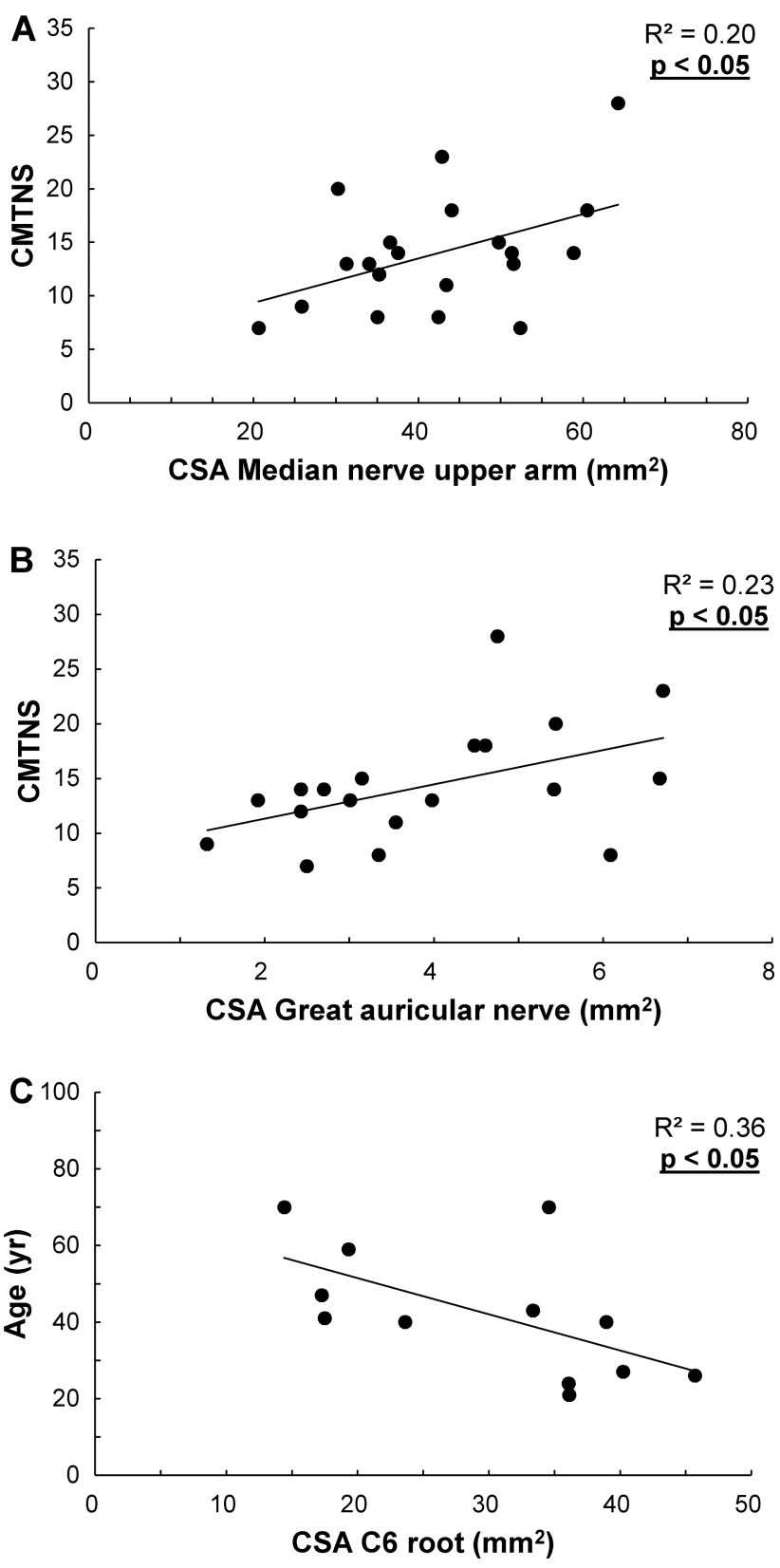

Figure 3 Scatterplot of the clinical parameters and ultrasound findings in patients with CMT1A. The CMTNS was positively correlated with the CSA of the great auricular nerve and that of the median nerve at the upper arm (A and $B)$. An inverse correlation between the $C 6$ root CSAs and age was observed (C). CMTNS, CMT neuropathy score; CSA, cross-sectional area; CMT, Charcot-Marie-Tooth disease.

activates the transcription of several myelin-associated genes, such as PMP22 and MPZ. Although we could include only one patient with EGR2-associated CMT, CSAs in all nerves tended to be larger than in controls. We also included the patient with CMT who had a potent novel mutation in the ARHGEF10 gene, as aforementioned. The phenotype of the patient was classified as the axonal type by neurophysiological testing, but the MCV was moderately slowed (median nerve MCV $41.7 \mathrm{~m} / \mathrm{s}$ ), as well as in previous studies. ${ }^{20} 21$ Verhoeven et al demonstrated the possibility that ARHGEF10 protein is associated with the developmental myelination of peripheral nerves using a mouse model. CSAs in all nerves were increased in the present patient, although CSAs of the C6 root and great auricular nerve were not recorded because of technical difficulties.
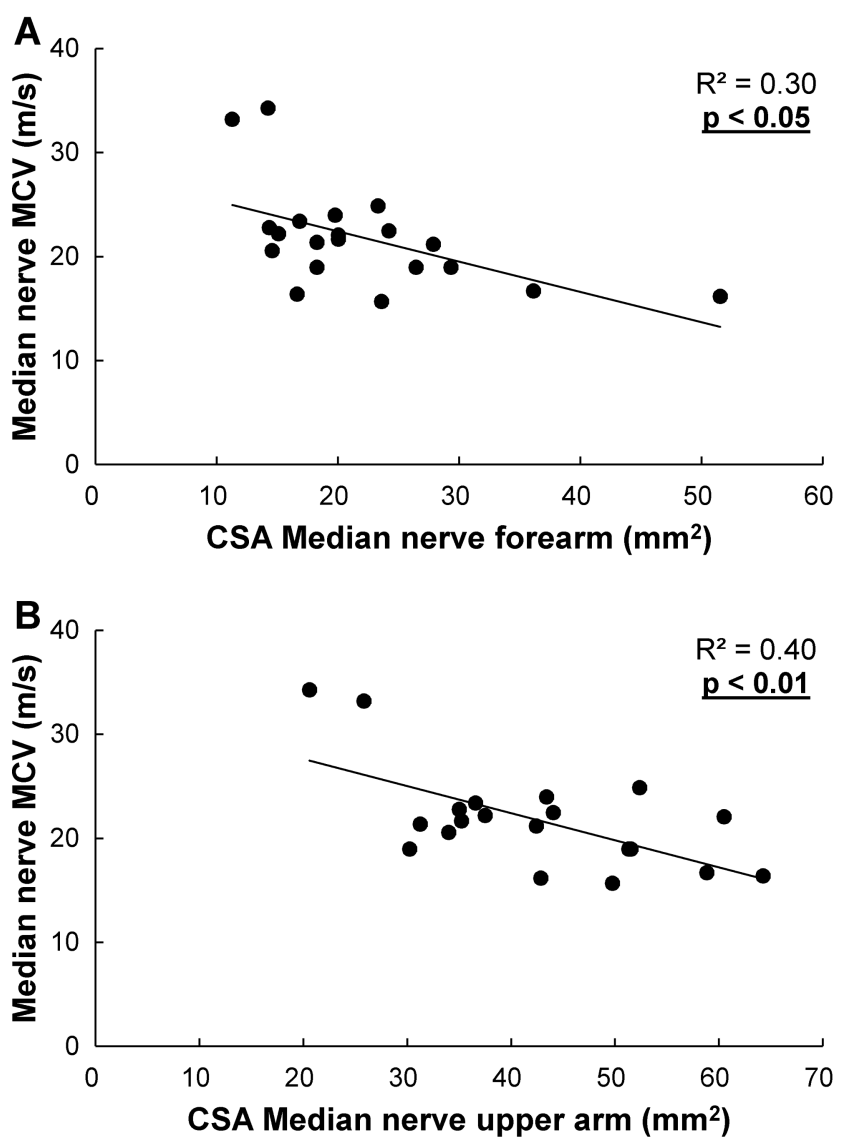

Figure 4 Scatterplot of the electrophysiological parameters and ultrasound findings in patients with CMT1A. A negative correlation between the CSA at the forearm and MCV of the median nerve (between the wrist and elbow) was found (A). Likewise the CSA at the upper arm of the median nerve was negatively correlated with the MCV of the median nerve (between the wrist and elbow) (B). CMT, Charcot-Marie-Tooth disease; CSA, cross-sectional area; MCV, motor conduction velocity.

Regarding the US findings of the patients with CMT1A, Pazzaglia et $a l^{15}$ demonstrated an inverse correlation between sural nerve CSAs and the age in patients with CMT1A. In this study, no such correlation was observed in patients with CMT1A. As aforementioned, the difference in the method of measuring CSAs could influence the results. Instead, a significant negative correlation between C6 root CSAs and the age was noted. However, there are some reports that the biometric data of patients with CMT showed no significant correlation with CSAs. $^{79}$ Thus, the results of correlation analysis between CSAs and biometric data have varied among reports. The reason for this remains unclear, and so further studies involving larger series of cases are needed.

This study first showed the correlation between the disease severity (CMTNS) and CSAs in patients with CMT1A. Patients with a larger CSA in the median or great auricular nerve may show more marked impairment. It is extrapolated from these results that the degree of the disease severity might be determined by the extent of the pathological change, such as onion bulbs which are the results of repetitive demyelination-remyelination and the proliferation of the nerve stroma. On the other hand, it seems that the positive relationship between CMTNS and CSAs contradicts the inverse relationship between age and CSAs in this study (figure 3), because CMTNS generally increases with age in patients with CMT. Future studies will be 
required to elucidate whether age or disease severity has more influence on the nerve enlargement in CMT1A. Along with the report by Pazzaglia $e t ~ a l,{ }^{15}$ the negative correlation between age and CSAs in most of the nerves might be specific to CMT1A, and indicates that decreased CSAs reflect axonal loss. Conversely, only CSA in median nerve at wrist correlated with age positively in patients with CMT1A of this study, although it was not statistically significant. CSA at wrist might be affected by factors except for CMT1A including carpal tunnel syndrome (CTS), etc. In patients with CTS, median nerve CSAs at wrist are generally increased. ${ }^{22}$

Several studies have reported on the relationships between US findings and NCS parameters in CMT and other neuropathies. ${ }^{7} 9$ 23-25 Consistent with a previous study by Schreiber et al, we identified a significant negative correlation between the CSAs of median nerve and the MCVs in the corresponding segment. The decreased MCVs in patients with CMT1A reflect the functional aspect for the histopathological alteration of myelination, progression of which might have paralleled the enlargement of nerves, that is, increased CSAs.

There are some limitations to our study. First, US examinations were performed by only one unblinded examiner. However, Cartwright $e t a l^{26} 27$ reported that the diagnostic accuracy of neuromuscular US in unblinded studies was similar to that in blinded studies, and that intra-rater and inter-rater reliability of nerve and muscle US were sufficiently high. This argument may mitigate the unblinded design in this study to some extent; however, blinded assessment by multiple examiners is desirable in future studies on nerve US. Second, the small number of some CMT types is also a limitation of our study. Therefore, the findings of CSAs obtained from a single or a few patients should be carefully interpreted. Further study of a large population is needed, especially in MPZ-associated and NEFL-associated CMT in which demyelinating and axonal types are mixed. Third, the US feature of CMT1A has been already revealed by some studies. ${ }^{5-7} 914$ However, describing the US finding of that was needed for shedding light on the extent of nerve enlargement in other rare CMT subtypes. In addition, nerve CSAs correlated with the clinical severity in CMT1A can provide a new insight into the evolving field of nerve US. Finally, our CSA measuring method including the hyperechoic rim is different from the method in most previous studies of nerve US with tracking inside the rim. Therefore, US findings in our study should be compared with other studies of nerve US with caution. However, our method might make it possible to assess the actual pathology of CMT because structures other than Schwann cells could proliferate in CMT1A. ${ }^{16}$

In conclusion, we have demonstrated US findings at diverse anatomical sites of patients with CMT subtypes. We confirmed the uniform enlargement of peripheral nerves in patients with CMT1A. We also found that patients with demyelinating-type CMT, such as CMT1F (NEFL-associated CMT), do not always exhibit nerve enlargement. Nerve US in addition to conventional NCS could facilitate targeted gene analysis in clinical situations, and may advance the understanding of peripheral nerve pathology in patients with CMT.

Contributions $\mathrm{Y}$-iN was involved in design of the study, analysis of the data and drafting of the manuscript. KS was involved in design of the study, acquisition and interpretation of data and revision of the manuscript. YT was involved in interpretation of the data. IM was involved in acquisition and analysis of the data. YH, AH and HT were involved in acquisition and analysis of the data and drafting of the manuscript. MN was involved in design of the study and revision of the manuscript.

Funding The work was partly funded by the Intramural Research Grant for Neurological and Psychiatric Disorders of NCNP, Applying Health and Technology of Ministry of Health,
Welfare and Labour, Japan and Grants-in-Aid from the Research Committee of CharcotMarie-Tooth Disease, the Ministry of Health, Labour and Welfare of Japan.

Competing interests None.

Ethics approval The local ethics committee of Kyoto Prefectural University of Medicine.

Provenance and peer review Not commissioned; externally peer reviewed.

\section{REFERENCES}

1 Harding AE, Thomas PK. The clinical features of hereditary motor and sensory neuropathy types I and II. Brain 1980;103:259-80.

2 Shy ME, Siskind C, Swan ER, et al. CMT1X phenotypes represent loss of GJB1 gene function. Neurology 2007;68:849-55.

3 Murphy SM, Laura M, Fawcett K, et al. Charcot-Marie-Tooth disease: frequency of genetic subtypes and guidelines for genetic testing. J Neurol Neurosurg Psychiatry 2012;83:706-10.

4 Hattori N, Yamamoto M, Yoshihara T, et al. Demyelinating and axonal features of Charcot-Marie-Tooth disease with mutations of myelin-related proteins (PMP22, MPZ and (X32): a clinicopathological study of 205 Japanese patients. Brain 2003; 126:134-51.

5 Heinemeyer 0 , Reimers CD. Ultrasound of radial, ulnar, median, and sciatic nerves in healthy subjects and patients with hereditary motor and sensory neuropathies. Ultrasound Med Biol 1999;25:481-5.

6 Zaidman CM, Al-Lozi M, Pestronk A. Peripheral nerve size in normals and patients with polyneuropathy: an ultrasound study. Muscle Nerve 2009;40:960-6.

7 Martinoli C, Schenone A, Bianchi S, et al. Sonography of the median nerve in Charcot-Marie-Tooth disease. AJR Am J Roentgenol 2002;178:1553-6.

8 Cartwright MS, Brown ME, Eulitt $P$, et al. Diagnostic nerve ultrasound in Charcot-Marie-Tooth disease type 1B. Muscle Nerve 2009;40:98-102.

9 Schreiber $S$, Oldag A, Kornblum C, et al. Sonography of the median nerve in CMT1A, CMT2A, CMTX, and HNPP. Muscle Nerve 2013;47:385-95.

10 Zhao Z, Hashiguchi A, Hu J, et al. Alanyl-tRNA synthetase mutation in a family with dominant distal hereditary motor neuropathy. Neurology 2012;78:1644-9.

11 Matsuoka N, Kohriyama T, Ochi K, et al. Detection of cervical nerve root hypertrophy by ultrasonography in chronic inflammatory demyelinating polyradiculoneuropathy. J Neurol Sci 2004;219:15-21.

12 Shy ME, Blake J, Krajewski K, et al. Reliability and validity of the CMT neuropathy score as a measure of disability. Neurology 2005;64:1209-14.

13 Shiga $K$, Noto Y, Mizuta I, et al. A novel EGR2 mutation within a family with a mild demyelinating form of Charcot-Marie-Tooth disease. J Peripher Nerv Syst 2012;17:206-9.

14 Sugimoto T, Ochi K, Hosomi N, et al. Ultrasonographic nerve enlargement of the median and ulnar nerves and the cervical nerve roots in patients with demyelinating Charcot-Marie-Tooth disease: distinction from patients with chronic inflammatory demyelinating polyneuropathy. J Neurol 2013;260:2580-7.

15 Pazzaglia C, Minciotti I, Coraci D, et al. Ultrasound assessment of sural nerve in Charcot-Marie-Tooth 1A neuropathy. Clin Neurophysiol 2013;124:1695-9.

16 Robaglia-Schlupp A, Pizant J, Norreel JC, et al. PMP22 overexpression causes dysmyelination in mice. Brain 2002;125:2213-21.

17 Fabrizi GM, Cavallaro T, Angiari C, et al. Charcot-Marie-Tooth disease type 2E, a disorder of the cytoskeleton. Brain 2007;130:394-403.

18 Miltenberger-Miltenyi G, Janecke AR, Wanschitz JV, et al. Clinical and electrophysiological features in Charcot-Marie-Tooth disease with mutations in the NEFL gene. Arch Neurol 2007;64:966-70.

19 Zaidman CM, Harms MB, Pestronk A. Ultrasound of inherited vs. acquired demyelinating polyneuropathies. J Neurol 2013;260:3115-21.

20 De Jonghe $\mathrm{P}$, Timmerman V, Nelis $\mathrm{E}$, et al. A novel type of hereditary motor and sensory neuropathy characterized by a mild phenotype. Arch Neurol 1999;56:1283-8.

21 Verhoeven $K$, De Jonghe $P$, Van de Putte $T$, et al. Slowed conduction and thin myelination of peripheral nerves associated with mutant rho Guanine-nucleotide exchange factor 10. Am J Hum Genet 2003;73:926-32.

22 Nakamichi K, Tachibana S. Ultrasonographic measurement of median nerve cross-sectional area in idiopathic carpal tunnel syndrome: diagnostic accuracy. Muscle Nerve 2002;26:798-803.

23 Scheidl E, Bohm J, Simo M, et al. Ultrasonography of MADSAM neuropathy: focal nerve enlargements at sites of existing and resolved conduction blocks. Neuromuscul Disord 2012;22:627-31.

24 Watanabe $\mathrm{T}$, Ito $\mathrm{H}$, Sekine $\mathrm{A}$, et al. Sonographic evaluation of the peripheral nerve in diabetic patients: the relationship between nerve conduction studies, echo intensity, and cross-sectional area. J Ultrasound Med 2010;29:697-708.

25 Tsukamoto H, Granata G, Coraci D, et al. Ultrasound and neurophysiological correlation in common fibular nerve conduction block at fibular head. Clin Neurophysiol 2014;125:1491-5.

26 Cartwright MS, Hobson-Webb LD, Boon AJ, et al. Evidence-based guideline: neuromuscular ultrasound for the diagnosis of carpal tunnel syndrome. Muscle Nerve 2012;46:287-93.

27 Cartwright MS, Demar S, Griffin LP, et al. Validity and reliability of nerve and muscle ultrasound. Muscle Nerve 2013:47:515-21. 